\title{
Propuesta didáctica para la enseñanza de las ecuaciones polinomiales
}

\author{
Prof.: Lic. Hernán Víquez Céspedes \\ Universidad de Costa Rica; hvc71287@gmail.com
}

Recibido: 09 de noviembre, 2016

\section{RESUMEN}

Este artículo contiene los principales resultados obtenidos a partir del diseño, aplicación y evaluación de una propuesta didáctica para la enseñanza de las ecuaciones polinomiales. La propuesta tiene como eje principal la Teoría del Aprendizaje por Competencias bajo el enfoque del Pensamiento Complejo, además toma aspectos específicos de distintas corrientes de la Didáctica Francesa, incorporando a su vez el uso de Tecnologías de la Información y la Comunicación (TIC). Los resultados de aprendizaje obtenidos con la implementación de la propuesta fueron muy satisfactorios, considerando que se pudo evidenciar una adquisición de conocimientos por parte de los estudiantes, así como una capacidad para integrar el tema de ecuaciones polinomiales con otros tópicos de las matemáticas.

Palabras clave: ecuaciones polinomiales, álgebra, TIC, pensamiento complejo, aprendizaje por competencias, didáctica francesa.

\section{ABSTRACT \\ DIDACTICAL PROPOSAL FOR TEACHING POLYNOMIAL EQUATIONS}

This article contains the main results obtained from the design, application and evaluation of a didactic proposal for the teaching of polynomial equations. The main thrust of the proposal is the Theory of Learning by Competencies under the Complex Thinking approach. It also takes specific aspects of different streams of French Didactics, incorporating the use of Information and Communication Technologies (ICT). The learning outcomes obtained with the implementation of the proposal were very satisfactory, considering that it was possible to evidence an acquisition of knowledge by the students, as well as an ability to integrate the theme of polynomial equations with other mathematical topics.

Key words: polynomial equations, algebra, ICT, complex thinking, competence learning, French didactics.
Aceptado: 17 de febrero, 2017

\section{Introducción}

La educación actual se enmarca en una sociedad donde la tecnología juega un rol fundamental en gran parte de las actividades que los individuos llevan a cabo. Es una herramienta que dinamiza el conocimiento y la información, lo cual exige nuevos sistemas educativos que preparen ciudadanos con la capacidad de afrontar los cambios emergentes. En particular, la educación matemática debe responder a esa necesidad, perfeccionando la práctica de metodologías tradicionales e incorporando nuevas estrategias didácticas donde la tecnología permita desarrollar en los estudiantes las habilidades requeridas. Actualmente, muchas de las investigaciones y propuestas planteadas para el tema de álgebra básica carecen de una fundamentación teórica y de una implementación supervisada que permita validar con éxito las herramientas didácticas que se involucran. A través de una revisión bibliográfica a nivel de América Latina, España, Estados Unidos y Canadá se constató dicho argumento.

Los resultados obtenidos, específicamente, en América Latina fueron reducidos, pues parece ser, que en los últimos años no se han implementado propuestas que contribuyan significativamente, con el aprendizaje del álgebra. Sin embargo, se pueden mencionar algunos sitios web, investigaciones y artículos que tratan el tema en cuestión.

A nivel nacional, se indagó el sitio web de la Revista Digital Matemática, Educación e Internet que proporciona el Instituto Tecnológico de Costa Rica1 ${ }^{1}$, el cual pone a disposición del

1. http://www.cidse.itcr.ac.cr/revistamate/index.htm 
docente una serie de laboratorios en formato Excel que permiten repasar en clase el concepto de ecuaciones de primer grado con una incógnita. Por otro lado, en la sección de software educativo se despliega una colección de applets (pequeños programas donde se pueden manipular ciertas condiciones y observar los resultados) para los estudiantes relacionados con el trazado de gráficas y la ubicación de puntos en el plano cartesiano.

En México y Canadá por su parte, se proponen los Sistemas Algebraicos Computarizados (SAC) (Kieran, 2005; Cedillo, 2006) mediante experiencias puestas en práctica con estudiantes y docentes, quienes manifiestan un aprendizaje significativo al incursionar en el uso de las calculadoras gráficas o cualquier otro programa que reciba lenguaje simbólico.

El investigador colombiano Castillo (2006) propone en su país un modelo para contribuir a desarrollar la habilidad de fundamentar-demostrar conceptos algebraicos en una clase de matemática basándose en siete tareas que deben desarrollar los docentes, algunas de ellas son: precisar los objetivos por indagar en el tema, indagar los conocimientos previos, elegir actividades de motivación, planear la sesión de clase, entre otras.

Es importante resaltar que dicho modelo hasta el momento de esta investigación, parecía no haber sido implementado ni validado en ninguna aula. No obstante, Acosta, Macías y La Red (2006, p.1) describen una propuesta desarrollada por la facultad de Ciencias Exactas y Naturales de la Universidad Nacional del Nordeste (Argentina), que consiste en dividir el curso Matemática I en dos modalidades: presencial y a distancia. Para lograr alcanzar los objetivos a distancia se diseña un software educativo llamado MaDiMaC, dicho software contiene las clases que comúnmente se imparten en la modalidad presencial, así como una serie de ejercicios con sus respectivas respuestas. Para los estudiantes matriculados en dicha modalidad, se abrieron foros de consulta en la web que permitían aclarar dudas y compartir experiencias de aprendizaje con otros compañeros (Acosta et al, 2006, p.2).
Cabe resaltar que la propuesta anterior no mostró resultados significativos, pues las notas obtenidas por los estudiantes matriculados en la modalidad presencial no variaron mucho en relación con las calificaciones obtenidas por los estudiantes matriculados en la modalidad a distancia (Acosta et al, 2006, p.4).

Por otra parte, el sitio web de la Federación Española de Profesores de Matemática ${ }^{2}$ presenta una serie de revistas que tratan temas interesantes de álgebra básica, sin embargo no estaban disponibles de momento, requerían afiliación mensual o los links direccionales estaban "rotos" por lo que no pudieron ser accedidas.

No obstante, el autor español Sierra (2010) presenta una serie de recursos para la enseñanza del álgebra, dentro de dichas herramientas se puede mencionar el Hot Potatoes, el cual permite desarrollar ejercicios matemáticos y publicarlos fácilmente en la red; el Wiris que corresponde a una plataforma virtual para ejecutar cálculos matemáticos; Ecuación ES que funciona como un tutor informático para resolver ecuaciones de segundo grado; el Proyecto Descartes, promovido por el Ministerio de Educación y Ciencia Española que presenta un entorno Java con una colección de applets matemáticos en todos los temas incluido el álgebra, entre otros.

En Estados Unidos y Canadá existen algunas tendencias en cuanto al desarrollo de propuestas para la enseñanza del álgebra básica. Entre ellas, se encuentra el uso de mosaicos (algebra tiles, en inglés) que consisten en una representación de expresiones algebraicas por medio de cuadriláteros, asignando a cada variable una medida y a la unidad se representa con un cuadrado pequeño. Así, será ilustrado con un cuadrado de lado y es un rectángulo de largo y ancho (Kilgore y Capraro, 2010, p. 118). Este tipo de representaciones, tienen como fin permitir al estudiante la manipulación directa de los objetos para facilitar la comprensión algebraica. Kilgore y Capraro (2010, p. 120) en su implementación involucran en sus clases una pizarra interactiva (SmartBoard) para explicar los procedimientos

2. http://www.fespm.es/-Revistas 
que sus estudiantes llevan a cabo con papel y lápiz o sus propios mosaicos. Sin embargo, esta metodología tiene el inconveniente de la limitación para representar polinomios de grados mayores a 3. Además, no se hallaron propuestas que contaran con una validación de la misma o al menos incluyera la evaluación del aprendizaje de los participantes.

Por otro lado, Laughbaum (2003a; 2003b; 2003c) propone la Enseñanza del Álgebra desde el Enfoque de Funciones en contraposición a su enseñanza por medio de ecuaciones, la cual pretende utilizar nociones básicas de funciones como la relación entre datos, las conexiones de representaciones numéricas, gráficas y simbólicas y el comportamiento de gráficas de funciones según el cambio de parámetros (Laughbaum, 2003a, p.2). Siguiendo esta línea, los polinomios y las ecuaciones desde un inicio están conectadas con situaciones de la vida cotidiana donde se dé la dependencia entre cantidades y no son simplemente objetos abstractos para el estudiante. Uno de los elementos fundamentales es el uso de calculadoras gráficas, ya que se busca analizar conceptos como ceros y grados de un polinomio, a partir de los gráficos de las respectivas funciones, aunque una opción sería reemplazar este instrumento por software libre que realice tareas similares.

Así mismo, existen propuestas de software desarrollados exclusivamente para la enseñanza del álgebra, como lo es el Cognitive Tutor Algebra, de la Universidad Carnegie Mellon y el Carnegie Learning, el cual tiene el propósito de fungir como una guía para los estudiantes, tal como se haría en una tutoría en la vida real, presentando ejercicios y orientando en caso de que existan dificultades y advirtiendo los errores cometidos (Ritter et al, 2007, p. 251). Se basa en la Teoría de la Cognición de Control Adaptativo del Pensamiento (ACT-R) (Ritter et al, 2007, p. 250) que busca crear tecnologías que interpreten la conducta humana con el fin de monitorear el progreso alcanzado con respecto a su capacidad cognitiva. Los autores recopilan una serie de investigaciones donde se evalúa la eficacia de esta herramienta, contrastándola con el uso del libro de texto y clases tradicionales y se ha evidenciado, en lo principal, un aumento en la autoconfianza de los estudiantes involucrados (2007, p. 252).

En el Laboratorio Leibniz de la Universidad Joseph Fourier, Grenoble, Francia, se ha creado el software Aplusix, dirigido a jóvenes de 12 a 16 años y elaborado como producto de investigaciones acerca del modelaje del razonamiento humano (Nicaud, Bouhineau, Varlet y NguyenXuan , 1999, p. 1) y se ha mejorado desde sus primeras aplicaciones piloto en 1987 hasta la fecha, añadiendo y reemplazando distintas opciones y entornos de tareas: práctica, micromundos, autoevaluación, entre otros. En su artículo, Nicaud et al. (1999) exponen los datos generados a partir de experimentos llevados a cabo en Italia, Brasil, India y Francia, donde Aplusix fue pieza central. A pesar de ciertas limitantes, se logró evidenciar una tendencia creciente hacia la autocorrección en los estudiantes y un incremento en su autoconfianza a la hora de trabajar individualmente (p. 87).

Por su parte, Goulding y Kyriacou (2008) investigadores británicos, presentan una revisión de investigaciones referentes a implementación de TIC en la enseñanza del álgebra a nivel de secundaria, incluyendo software y hardware. Los estudios que abarcaron desde 1996 a 2006, se agruparon en categorías, en una de ellas, referente a las ganancias en la comprensión, se reflejó que estudiantes que trabajaron con medios computacionales presentaron un mejor desempeño que aquellos que usaron lápiz y papel (Sivasubramaniam, 2000, citado por Goulding y Kyriacou 2008, p. 13), así como en otros casos no hubo diferencias destacables. (Isiksal y Askar, 2005, citado por Goulding y Kyriacou, 2008, p. 13).

Finalmente, con respecto al uso de GeoGebra como un recurso didáctico en la clases de álgebra, es posible encontrar en Internet manipulables (hojas dinámicas, generalmente con gráficas con las cuales los y las estudiantes puede interactuar y modificar) diseñados con este fin, sin embargo son limitadas las propuestas que incluyen una validación o respaldo teórico. $\mathrm{Lu}$ (2009) expone los resultados de su estudio exploratorio para conocer las percepciones de 
docentes de secundaria en Inglaterra y Taiwán acerca de este software como herramienta para el aprendizaje de la materia, las entrevistas realizadas dejan ver que, mientras en Inglaterra los profesores tenían una actitud positiva y sacaban mayor provecho a las actividades con GeoGebra, en Taiwán, probablemente por las dificultades de infraestructura, sólo se usaba para ilustrar ejemplos y ejercicios (p.65).

\section{Referente Teórico}

Para garantizar la validez del trabajo, se tomaron como referencia elementos de diversas teorías que sirven como fundamentación. La Teoría de la Transposición Didáctica, aporta la noción del sistema educativo como una triada, donde interactúan el o la docente, el o la alumna y el saber, el cual debe ser "transpuesto" para pasar de saber sabio a saber a enseñar y de ahí a saber enseñado (Chevallard, 1997). Es un proceso en el que convergen varios actores (científicos, especialistas en educación, miembros de la sociedad, entre otros) y que debe tomar las características de los estudiantes y de la sociedad en la cual se desarrollan.

En primer lugar, las transformaciones que sufra el saber sabio no tienen que afectar su contenido en sí, en el sentido en que se mantenga matemáticamente aceptable y no banalizado, pues si esto sucede se cae en lo que se conoce como desgaste biológico, que también se refiere a cuando algún elemento del saber deja de ser válido para la comunidad científica (se demuestra falso, por ejemplo). Además, existe otro tipo de desgaste llamado desgaste moral, básicamente ocurre cuando se desfasa lo enseñado con el desarrollo sociocultural (Chevallard, 1997). En otras palabras, el conocimiento que se manifiesta en los salones de clase tiene que actualizarse, de ahí que en este trabajo se busque incorporar el uso de TIC en la enseñanza de la matemática.

El uso de TIC responde también al objetivo de diseñar actividades de aprendizaje acordes con lo que propone la Teoría de Situaciones Didácticas (Brousseau 1986), donde se permita al estudiante enfrentarse a situaciones que le provoquen un desequilibrio y una necesidad implícita por construir un nuevo conocimiento para poder superarlas.

Esto es lo que se conoce como situación adidáctica, que a diferencia de una situación de la vida cotidiana en la cual el estudiante debería adaptarse naturalmente, aquí existe una intencionalidad didáctica. Se supone que el o la docente, a pesar de ser quien propone la actividad, no toma un papel activo si no hasta una vez concluida, momento en el que se realiza el proceso de institucionalización del saber, es decir, donde los conocimientos adquieren un estatuto de contenido matemático.

Basándose en lo anterior, se puede mencionar que las demandas sociales apuntan, actualmente, al desarrollo de una era tecnológica que le permita a los nuevos ciudadanos interactuar y transformar su entorno (Tobón 2004, p.20), y qué mejor manera de hacerlo que incorporar las nuevas tecnologías al salón de clase y guiar a los futuros profesionales por una vía socialmente adecuada, que al parecer es incierta y cambiante.

Brousseau (1986), el principal exponente de la Teoría de Situaciones Didácticas, señala algunos efectos y paradojas de aprendizaje que pueden generarse al emplear las situaciones didácticas de forma incorrecta (Brousseau, 1986, p. 25). Entre ellas está el Efecto Topace, cuando el o la docente intenta ayudar al estudiante a llegar a una conclusión dándole pistas o información que hacen evidente el conocimiento que debería extraer por sí mismo. Otro, es el Deslizamiento Metacognitivo que se da al enfocar como objeto de estudio alguno de los elementos que sirven de medio para alcanzar el conocimiento. Muestra de esto sucede al incurrir en el error de presentar las herramientas tecnológicas como un fin de estudio, y no como un facilitador.

No obstante, la noción de situación didáctica guarda relación, aunque no ha de confundirse, con el concepto de situación planteado por Vergnaud (1990) al referirse a los Campos Conceptuales. Según Vergnaud, cuando el individuo lleva a cabo actividades matemáticas, se localiza en un campo conceptual, es decir un conjunto de problemas donde se involucran conceptos, procedimientos y representaciones que no son las mismas pero sí guardan una estrecha 
relación (p. 135). Así, por ejemplo, al sumar, multiplicar y dividir polinomios se requiere manejar procedimientos análogos a los que se emplearían al efectuar las mismas operaciones con números enteros. En particular, el algoritmo de la división para polinomios es prácticamente el mismo que el algoritmo de la división en los enteros.

Por otra parte, es importante resaltar que para lograr el desarrollo profesional en sociedad es necesario que el estudiante adquiera una serie de competencias que le permitan enfrentar los procesos de incertidumbre que caracterizan el diario vivir (Margery, 2010) la teoría del Aprendizaje por Competencias y del Pensamiento Complejo vienen a dar el sustento teórico necesario para este tipo de temas. Según Tobón, una competencia desde el punto de vista del pensamiento complejo se entiende como un

(... ) proceso complejo de desempeño con idoneidad en determinados contextos, integrando diferentes saberes, para realizar actividades y/o resolver problemas con sentido de reto, motivación, flexibilidad, creatividad, comprensión y emprendimiento, dentro de una perspectiva de procesamiento metacognitivo, mejoramiento continuo y compromiso ético, con la meta de contribuir al desarrollo personal, la construcción y afianciamiento del tejido social, la búsqueda continua del desarrollo económico empresarial sostenible, y el cuidado y protección del ambiente y de las especies vivas (Tobón, 2008, p.5).

Las competencias que adquieren los estudiantes durante su proceso de formación académica no son únicas, varían dependiendo de la etapa escolar en la que se encuentre el futuro profesional; por ejemplo, las competencias adquiridas por los niños y los adolescentes en la primaria y la secundaria se clasifican como básicas, pues sólo aportan las habilidades para enfrentar los problemas de la vida cotidiana, no obstante, a nivel superior se adquieren competencias genéricas y específicas, las cuales se refieren a las competencias compartidas de cada profesión, así como, a las que son propias de cada área del saber (Tobón, 2008). También hay quienes adquieren las llamadas competencias transversales, las cuales son comunes a un grupo determinado de carreras profesionales, como por ejemplo educación, medicina, administración, entre otros. Ahora bien, para que el estudiante logre alcanzar este tipo de habilidades a lo largo de su formación es necesario desarrollar ambientes educativos donde la prioridad no sea el producto final de una tarea asignada, sino más bien, el proceso de construcción del conocimiento de forma integral (no aislada) (Margery, 2010, p 32) que se pueda adquirir en la elaboración de dicha tarea. Es aquí donde entra en juego la teoría del pensamiento complejo, la cual, apoyándose en las competencias profesionales, le aportan al individuo las herramientas básicas requeridas para enfrentar la compleja sociedad en la cual se desenvuelve.

No obstante, si se quiere determinar el grado de adquisición de una competencia es necesario definir una serie de niveles de logro basados en un conjunto de habilidades que se esperaría el estudiante alcance en cada nivel, a estas competencias se les conoce como criterios de desempeño, los cuales permiten ubicar a un estudiante en un nivel determinado.

\section{Diseño e implementación de la propuesta didáctica}

A continuación se detalla la propuesta didáctica para la enseñanza de las ecuaciones polinomiales, tomando en consideración aspectos importantes que se desarrollaron durante la implementación de la misma, así como los resultados finales obtenidos.

La propuesta fue aplicada en el segundo semestre del año 2011 a un grupo de estudiantes del curso MA0125 Matemática Elemental de la Universidad de Costa Rica, Sede Rodrigo Facio, la matrícula constaba de 36 estudiantes de los cuales asistían a clases un promedio de 30 . El horario se desarrolló los lunes y jueves de 13:00 a 15:50 horas, la modalidad era teórica con una carga académica de 2 créditos.

El tiempo real de implementación fue de tres lecciones de 180 minutos cada una, no obstante se había planeado para desarrollarse en cuatro lecciones, sin embargo la coincidencia 
de un día feriado obligó a reducir la cantidad de clases. Cada lección contó con distintas actividades, incluyendo explicaciones magistrales de la docente pero dando un papel protagónico al trabajo individual y colectivo.

Por otra parte, la competencia que se desarrolló en esta propuesta estaba orientada a que el estudiante resolviera ecuaciones polinomiales con coeficientes racionales en una variable con base en los procedimientos desarrollados en el curso MA0125 Matemática Elemental de la Universidad de Costa Rica.

Dicha competencia se define como la capacidad de aplicar métodos de factorización de polinomios y operaciones algebraicas básicas para la resolución de ecuaciones polinomiales en una variable. Dado que el curso descrito está dirigido a estudiantes de diferentes carreras profesionales se decidió clasificar la competencia como transversal, ya que comprende habilidades que deben ser dominadas en diferentes áreas del saber.

Ahora bien, para la competencia planteada se diseñaron seis criterios de desempeño y tres niveles de logro que permitieron evaluar a los diferentes estudiantes y poder ubicarlos en un orden jerárquico con respecto al avance mostrado a lo largo de la implementación.

Los criterios de desempeño construidos fueron los siguientes:

1. Conceptualizar las nociones de polinomio y ecuación.

2. Interpretar la representación gráfica de polinomios de variable real.

3. Localizar puntos en el plano a partir de un polinomio y su valor numérico para números reales determinados.

4. Resolver operaciones con polinomios.

5. Factorizar polinomios mediante: factor común, inspección, fórmula general, división sintética y agrupación.

6. Determinar el conjunto solución de una ecuación polinomial con coeficientes racionales en una variable.

Basándose en estos criterios de desempeño se pudo ubicar a los estudiantes en uno de los siguientes niveles de logro construidos: básico, intermedio y estratégico. Cada uno de estos niveles está compuesto por una serie de características que permiten evidenciar el logro de la competencia por parte de los estudiantes.

A continuación se dan las características de los niveles de logro definidos.

\section{Nivel básico:}

- Expresa nociones intuitivas de polinomios y de ecuaciones.

- Localiza puntos en el plano a partir de un polinomio y su valor numérico para números reales determinados.

- Resuelve operaciones con polinomios que involucren: suma y resta.

- Factoriza polinomios mediante factor común e inspección de trinomios cuadráticos.

- Determina el conjunto solución de ecuaciones polinomiales que se resuelven por despeje directo.

\section{Nivel intermedio:}

- Conceptualiza las nociones de polinomio y ecuación.

- Deduce con apoyo los ceros de un polinomio y el conjunto solución de una ecuación polinomial a partir de la representación gráfica.

- Resuelve operaciones con polinomios que involucren suma y resta.

- Resuelve con apoyo operaciones con polinomios que involucren multiplicación y/o división.

- Factoriza polinomios mediante: factor común, inspección, fórmula general, división sintética y agrupación con apoyo para elegir el método apropiado.

- Determina el conjunto solución de ecuaciones lineales y cuadráticas.

- Determina con apoyo el conjunto solución de ecuaciones polinomiales de grado mayor o igual a 3 .

\section{Nivel estratégico:}

- Conceptualiza las nociones de polinomio y ecuación.

- Deduce los ceros de un polinomio y el conjunto solución de una ecuación polinomial a partir de la representación gráfica. 
- Resuelve operaciones con polinomios.

- Factoriza polinomios mediante: factor común, inspección, fórmula general, división sintética y agrupación.

- Determina el conjunto solución de una ecuación polinomial con coeficientes racionales en una variable.

- Justifica sus resultados con razonamientos matemáticos válidos.

\section{Actividades desarrolladas}

Dentro de las evidencias de aprendizaje utilizadas en la implementación se pueden nombrar el trabajo en clase, en el cual se efectuaron exposiciones y prácticas por parte de los estudiantes; tareas que involucran TIC, para ello se utilizan recursos como la plataforma de Moodle "Mediación Virtual", que pone a disposición la Unidad de Apoyo a la Docencia Mediada con Tecnologías de la Información y la Comunicación (METICS) de la Vicerrectoría de Docencia de la Universidad de Costa Rica, y el programa de software libre GeoGebra para desarrollar manipulables y tareas escritas que los estudiantes desarrollaban fuera del aula y que involucraban los contenidos vistos en clase.

Uno de los aspectos más importantes de la implementación es que se diseñó de manera tal que el desarrollo de la competencia se llevó a cabo en espiral, es decir, los estudiantes no aprendían habilidades aisladas, sino que desde un inicio se presentó el problema de resolver ecuaciones con polinomios, comenzando por las de primer grado y conforme se aumentaba el grado, y por ende la complejidad de las mismas, se introdujeron otras habilidades como resolver operaciones con polinomios y posteriormente la factorización.

Ahora bien, uno de los aportes en el aprendizaje de las ecuaciones polinomiales que conllevó el uso de TIC fue el poder incorporar la representación gráfica de polinomios a su aprendizaje, algo que típicamente no se realiza en la mayoría de los cursos similares al de Matemática Elemental. De esta forma, fue posible asociar conceptos como el de intersección de dos gráficas como solución de una ecuación, o el grado de un polinomio con la cantidad máxima de intersecciones de su gráfica con el eje X.

En una de las actividades iniciales, los estudiantes efectuaron una hoja de trabajo en la cual debían calcular el valor numérico de un polinomio y asociar el par ordenado correspondiente con el punto en el plano cartesiano. En la Figura 1 se ilustra este hecho, donde a través de GeoGebra se dan valores numéricos en los puntos rojos, y se va haciendo el trazado de la curva polinómica. Debido a que no se esperaba que los estudiantes tuvieran la capacidad de trazar gráficas de polinomios de grado mayor que 2, lo cual tampoco estaba dentro de los objetivos del curso, se utilizaron manipulables creados con GeoGebra para presentar las gráficas y permitir a los estudiantes analizar ciertas características. Tal es el caso de la actividad de la Figura 2 que se colocó en la plataforma de Moodle para que los estudiantes la realizaran individualmente. Los deslizadores del lado izquierdo permiten asignar los valores a cada uno de los coeficientes y así se construye la ecuación indicada, dando una sensación de movimiento en que el estudiante puede apreciar la dependencia que tiene la curva de los parámetros usados.

En la propuesta se incorporó también el uso

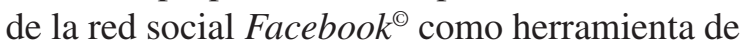
aprendizaje, específicamente, como medio para comunicarse con los estudiantes fuera de clase, a través de la creación de un Grupo ${ }^{3}$. Esta utilización se hizo de una forma experimental pues eran pocos los antecedentes que se tenían al respecto. En general, hubo una buena aceptación por parte de los estudiantes, quienes se matricularon en un $53 \%$ aproximadamente y en su mayoría lo aprovecharon para aclarar dudas acerca de la materia y el uso de la plataforma de Mediación Virtual (ver Figura 3). Las interacciones con la docente son principalmente para aclarar dudas sobre la materia o sobre los procedimientos para resolver ejercicios. También, se pudieron observar algunas interacciones entre los estudiantes,

3. Un grupo de Facebook permite que los usuarios inscritos en él publiquen comentarios, fotografías y enlaces entre ellos. 


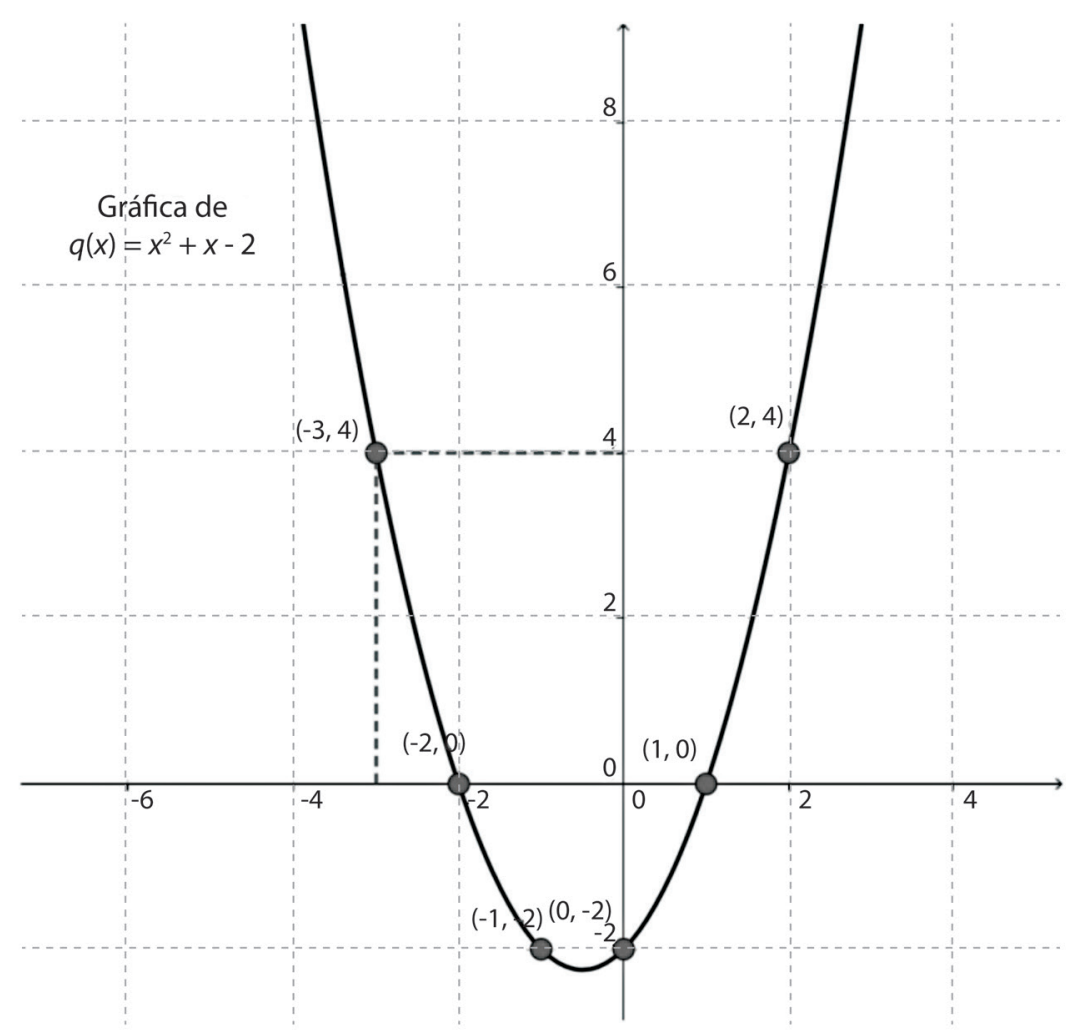

Figura No. 1. Gráfica de $q(x)=x^{2}+x-2$.

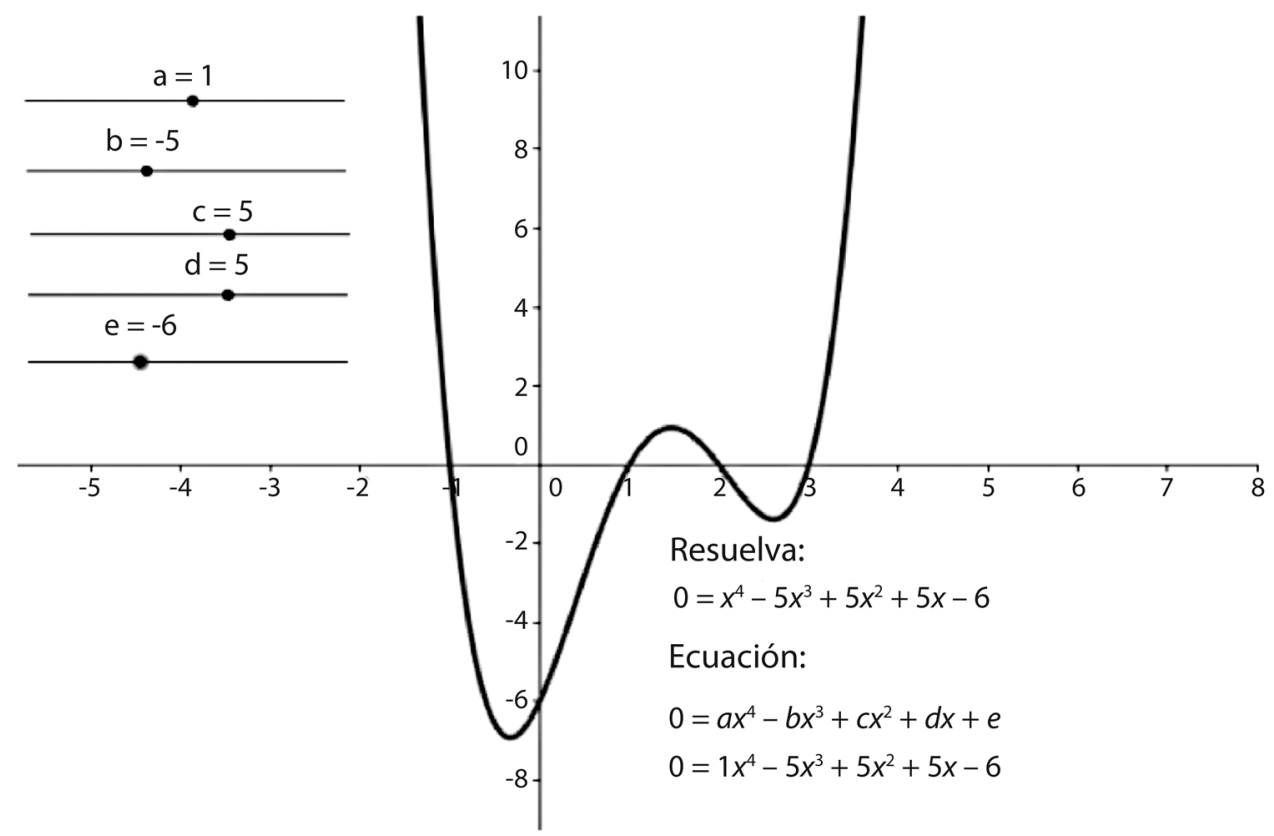

Figura No. 2. Manipulable desarrollado con GeoGebra. 
tengo otro problema con el ejercio \#9 de la tarea 1:

pide un factor del polinomio $(2 y-x)^{\wedge} 3-x(2 y-x)^{\wedge} 2$
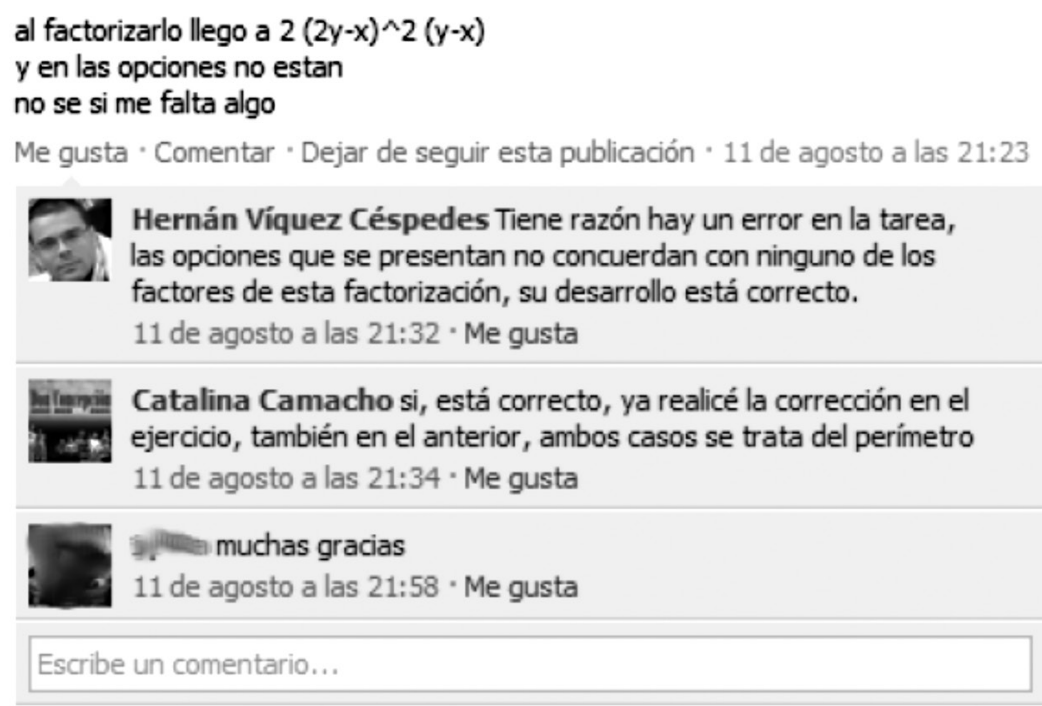

Figura No. 3. Dudas planteadas por estudiantes en el grupo de Facebook.

en que algunos que ya habían resuelto los ejercicios comentaban sus procedimientos a algún compañero que hacía una consulta.

Con respecto a la evaluación del aprendizaje, se tomaron en cuenta una prueba corta y una tarea. Debido a limitantes del tiempo sólo fue posible evaluar los criterios 1, 4, 5 y 6. Cabe mencionar que, por disposición de la cátedra, un $20 \%$ de la nota estaba asignada a las pruebas cortas, en este caso se obtuvo la autorización para que el porcentaje correspondiente a la primera prueba se dividiera en una parte desarrollada de forma presencial y dicha tarea. En la Tabla 1 se resumen los resultados de la evaluación. Tal y como se observa, para el criterio cuatro se generaron mejores resultados en la primera evaluación, pero para los criterios 5 y 6 y en la calificación final para la competencia, sucedió lo contrario. La causa de ello es probablemente que la prueba corta se realizó más próxima al momento en que se cubrió el tema en las lecciones. Por otro lado, en general el nivel de la competencia alcanzado fue de un 2.08, es decir en promedio los estudiantes se ubicaron en un nivel intermedio.

\section{Análisis de resultados}

Para el análisis de los resultados se tomaron en consideración las evaluaciones realizadas

TABLA No. 1.

Resumen de resultados de la evaluación de la competencia.

\begin{tabular}{lccccc}
\multicolumn{1}{r}{ Evaluación } & Criterio 1 & Criterio 4 & Criterio 5 & Criterio 6 & Competencia \\
Prueba Corta & 2,12 & 2,20 & 1,29 & 2,37 & 1,99 \\
Tarea & No aplica & 1,59 & 2,14 & 2,50 & 2,08 \\
General & 2,12 & 1,72 & 2,08 & 2,40 & 2,08
\end{tabular}

Fuente: elaboración propia. 
por los estudiantes dentro y fuera de clase, entre ellas se pueden rescatar una hoja de trabajo, una tarea, una prueba corta y la información recopilada acerca del desempeño de los estudiantes y de su respuesta al uso de TIC en el aula de matemática.

Se hace además, una comparación de los estudiantes sometidos a la propuesta y dos grupos control con características similares, esto con respecto a las primeras tres preguntas del primer examen parcial del curso en donde se evidencian tres de los criterios de desempeño construidos para la competencia. El análisis de los resultados giró en torno a cuatro ejes, el primero de ellos corresponde al análisis del logro de la competencia, aquí se desarrolló un estudio de desviación estándar para visualizar si la relación existente entre la información obtenida es alta, o por el contrario, es poco significativa. De la recopilación de información se pudo deducir que existe una amplia homogeneidad entre los datos ya que la desviación estándar para cada una de las variables (criterio 1 , criterio 4 , criterio 5 , criterio 6 y la competencia en general) es relativamente baja con respecto al promedio. Este análisis permitió ubicar a la mayoría de los estudiantes en el nivel de logro de la competencia número 2 para cada uno de los criterios estudiados, sin dejar de lado que existe una considerable población ubicada en el nivel 1 para el criterio 4 .

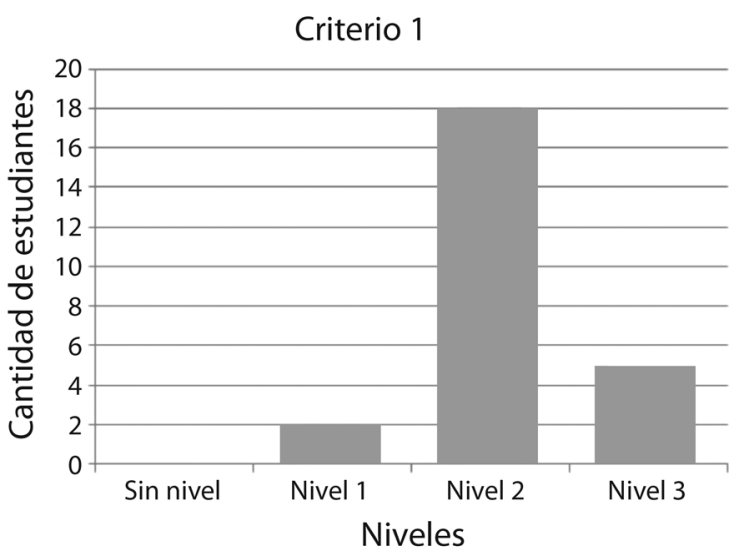

Gráfico No. 1.

Criterio 1 para la evaluación de la competencia. Fuente: elaboración propia.

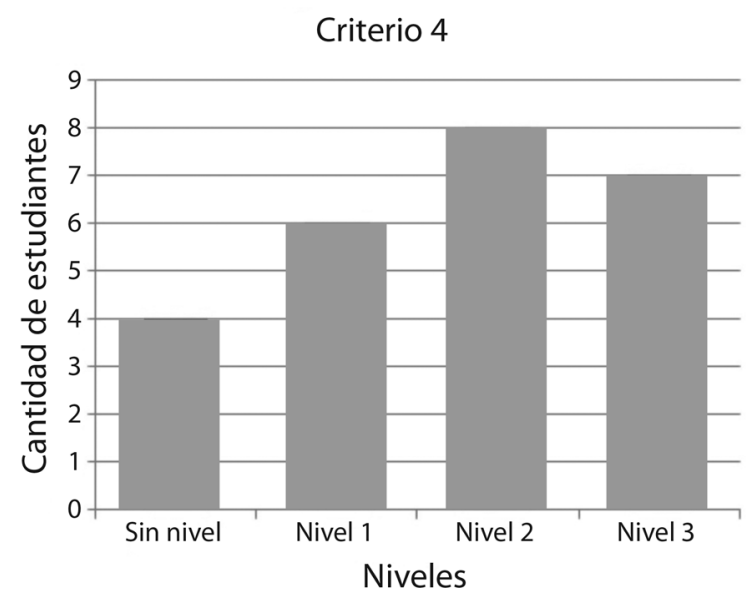

Gráfico No. 2.

Criterio 4 para la evaluación de la competencia. Fuente: elaboración propia.

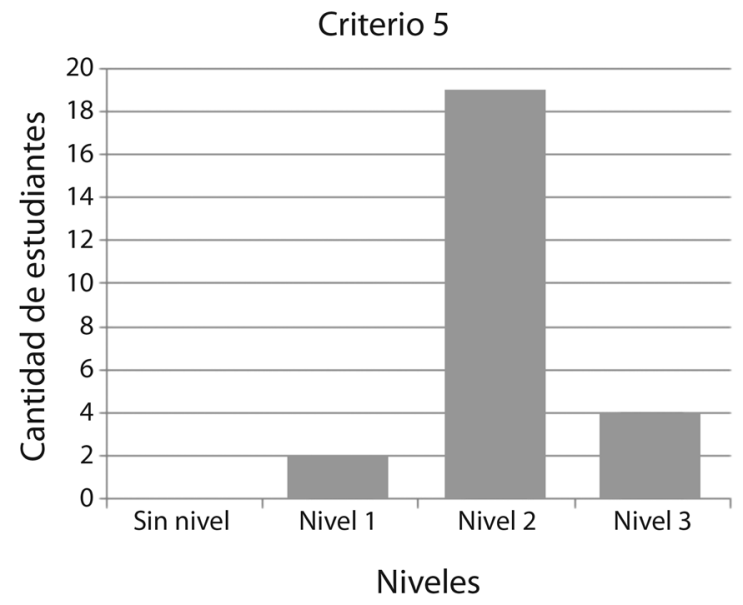

Gráfico No. 3.

Criterio 5 para la evaluación de la competencia. Fuente: elaboración propia.

Por último, la mayoría de los estudiantes sometidos a la propuesta se ubicaron en el nivel 2 de la competencia en general. También se desarrolló un análisis del logro de los criterios, plasmando la información obtenida en gráficas de barras que contienen las frecuencias de estudiantes para cada nivel de logro, ver Gráficos 1, 2, 3 y 4 . Se puede observar la relación existente entre el criterio de desempeño 1 y el criterio de desempeño 5, pues los datos de ambos están distribuidos de manera similar. El criterio 4 por 


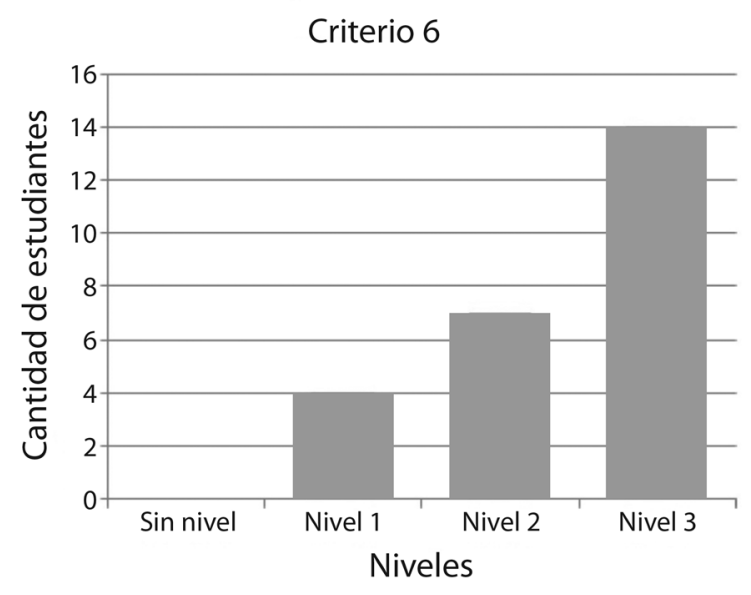

Gráfico No. 4.

Criterio 6 para la evaluación de la competencia. Fuente: elaboración propia.

ejemplo, es el único que posee estudiantes que no lograron alcanzar ningún nivel y es el único que presenta una distribución equitativa de estudiantes ubicados en los tres niveles de logro. El criterio 6 por su parte, es el que presenta más cantidad de estudiantes en el nivel 3.

El segundo eje del análisis de resultados desarrolló una comparación con los grupos control, hay que recordar que estos grupos no participaron de la propuesta, sino que recibieron lecciones de forma tradicional. Se realizó una prueba de hipótesis para el promedio general en cada ítem de los grupos de control (31 estudiantes) y el obtenido por el grupo experimental (28 estudiantes), se comprobó que, al establecer un nivel de significancia del 5\%, la diferencia entre ambos es prácticamente nula (aunque cabe aclarar que esta similitud no es tan marcada en la segunda pregunta, para la cual la probabilidad de error es poco más del $6 \%$ ), por lo que en realidad no parece haber una marcado influencia en el desempeño con respecto a la participación o no de los estudiantes en la propuesta y la injerencia de este factor en los resultados de la prueba.

\section{Conclusiones}

A continuación se presentan las principales conclusiones extraídas a partir del trabajo que implicó el diseño, aplicación y validación de la propuesta didáctica. En primer lugar, a través de la revisión bibliográfica se pudo evidenciar, principalmente en América Latina, una escasez de trabajos para la enseñanza y aprendizaje del álgebra que cuenten con una fundamentación teórica y/o validación posterior de su efectividad, pues en muchos casos únicamente se describen las propuestas sin haber sido aplicadas.

La elaboración de propuestas de este tipo, donde se integra un enfoque por competencias con el uso de TIC, requiere un planeamiento especializado y por ende, de una inversión de tiempo mayor, lo cual es un aspecto que se debe tomar en cuenta por parte del docente; sin embargo permite que el desarrollo de las lecciones se agilice. Además, un factor importante que se genera a partir del uso de TIC en el aula y fuera de ella es que se fomenta la participación de los estudiantes al trabajar con medios interactivos. También, se fortalece la comunicación estudiante-docente y de los estudiantes entre sí, por ejemplo a través de la utilización de Facebook.

Otro elemento que aporta la incorporación de las TIC en la enseñanza y aprendizaje de la matemática es que amplía la gama de representaciones de los objetos de estudio, y así permite una mejor compresión de los mismos, considerando que los estudiantes tienen distintas formas de aprender, y de comprender los conceptos.

Por su parte, el desarrollo de propuestas bajo el enfoque del pensamiento complejo, al considerar el conocimiento como un todo, hace necesario establecer conexiones entre distintas áreas de las matemáticas, tal es el caso de las nociones de funciones utilizadas en la propuesta para introducir el concepto de polinomios. Aunque este tipo de relaciones son inherentes a la materia, por lo general no son evidenciadas y los estudiantes las perciben como conocimientos aislados. Esto se complementó al implementar el aprendizaje en espiral.

Si bien es cierto que esta propuesta didáctica requiere nuevas implementaciones para observar su eficacia y mejorarla con el tiempo, consideramos valiosa la experiencia pues permite desarrollar cursos de matemática universitaria con un enfoque diferente que, según nuestra opinión, es más rico que el tradicional. 
Una recomendación que surge a raíz de los resultados de este trabajo es que el tiempo de implementación sea de mediano a largo plazo, pues los elementos involucrados deben tratarse de manera integral y no fraccionada. Por último, se considera importante que existan más trabajos bajo esta misma línea, donde se cuente con fundamentos teóricos y sean los mismos docentes quienes a través de la reflexión sobre su propia práctica lleven a cabo una evaluación y validación de sus propuestas.

\section{Referencias Bibliográficas}

Acosta, Julio César, Macías, Dora y La Red Martínez, David. (2005). El uso de las TICs en la enseñanza y aprendizaje de Matemática I. Argentina, Universidad Nacional del Nordeste. Recuperado de http://exa.unne. edu.ar/depar/areas/informatica/SistemasOperativos/ MADIMACVIEncuentroMat.pd

Brousseau, Guy. (1986). Fundaments and methods of didactique, en: G. Brousseau, N. Balacheff, M. Cooper, R. Sutherland y V. Warfield (Eds.) Theory Of Didactical Situations in Mathematics (pp 21-76). Kluwer Academic Publishers.

Castillo, Luis. (2006). El estudio de los conceptos matemáticos: enseñanza y aprendizaje en la educación tecnológica. Recuperado de http://www.pmfs.edu.co/new/ images/escuelaingenieria/archivos/produccion $\% 20$ docente/PROYECTO_AULA.pdf

Cedillo Ávalos, Tenoch Esaú. (2006). La Enseñanza de las Matemáticas en la Escuela Secundaria. Los Sistemas Algebraicos Computarizados. Revista Mexicana de Investigación Educativa, 11(28), 129-153. Recuperado de http://www.redalyc.org/pdf/140/14002807.pdf

Chevallard, Yves. (1997). La Transposición Didáctica: del Saber Sabio al Saber Enseñado. Buenos Aires: AIQUE.

Goulding Maria y Kyriacou, Chris. (2008). A systematic review of the use of ICTs in developing pupils understanding of algebraic ideas (Reporte $\mathrm{N}^{\circ} 1606 \mathrm{R}$ ) Recuperado de http://eppi.ioe.ac.uk/cms/LinkClick.as px?fileticket $=$ m8ipuZyhunE\%3d\&tabid=2380\&langu age=en-US. Londres: University of London,Institute of Education.
Kieran, Carolyn y Saldanha, Luis. (2005) "Computer algebra system (CAS) as a tool for coaxing the emergence of reasoning about equivalence of algebraic expressions". En Helen L. Chick, Jill L, Vincent (Eds.) Proceedings of 29th PME(Vol 3, 193-200). Recuperado de http://www.emis.de/proceedings/ PME29/PME29CompleteProc/PME29Vol3Fug_ Mou.pdf

Kilgore, Kelly y Capraro, Mary Margaret. (2010). A technological aproach to teaching factorization. Journal of Mathematics Education3(2), 115-125, Recuperado de http://educationforatoz.com/images/9.Kelly_E._ Kilgore_Mary_Margaret_Capraro.pdf

Laughbaum, Edward. (2003a). Function Approach to Algebra \& Implementation Through. The Ohio State University. Recuperado de http://www.math.osu. edu/ laughbaum.6/amatyc/2002/2002_algebra_ using_function_approach_implementation.pdf

Laughbaum, Edward. (2003b). Developmental algebra with function as the underlying theme, Preprint, Recuperado de http://www.math.osu.edu/ laughbaum.6/papers/ dev_alg_with_function_as_underlying_theme.pdf

Laughbaum, Edward. (2003c). A teaching lesson on factoring polynomials with technology. Ponencia presentada en XV Annual International $\mathrm{T}^{3}$ Conference, Nashville, Tennessee. Recuperado de http://www. math.osu.edu/ elaughba/presentations/2003_intl_t3_ conf_paper.pdf

Lu, Yu-Wen Allison. (2009). Linking geometry and algebra: English and Taiwanese upper secondary teachers' Approaches to the use of GeoGebra. Proceedings of the British Society for Research into Learning Mathematics, 29(1), 61-66. Recuperado de http://www.bsrlm.org.uk/IPs/ip29-1/BSRLMIP-29-1-11.pdf

Malo, Salvador, Fortes, Mauricio, Verdejo, Pilar y Orta, Magdalena. (2008). Propuestas y acciones universitarias para la transformación de la educación superior en América Latina: Informe final del Proyecto 6x4 UEALC. México, Aseguramiento de la Calidad en la Educación y en el Trabajo, S.C.

Margery, Enrique. (2010). Complejidad, Transdisciplinariedad y Competencias: Cinco Viñetas Pedagógicas. San José, Costa Rica: URUK Editores.

Nicaud, Jean-François, Bouhineau, Denis, Bronner, Alain, Mezerette, Sophie y Chaachoua, Hamid. (2005). 
Computer assisted assessment in elementary algebra, Recuperado en: http://hal.archives-ouvertes. fr/docs/00/19/04/00/PDF/Bouhineau-Denis-2005Aplusix-CAA.pdf. Grenoble: Université Joseph Fourier, Laboratoire Leibniz

Nicaud, Jean-François, Bittar, Marilena, Chaachoua, Hamid, Inamdar, Parimala y Maffei, Laura. (2005). Experiments with Aplusix in four countries. International Journal for Technology in Mathematics Education 13(2), 79-88. Recuperado de http://www.eric.ed.gov/ERICWebPortal/search/ detailmini.jsp?_nfpb=true\&_\&ERICExtSearch_ SearchValue_0=EJ $874205 \&$ ERICExtSear ch_SearchType_0=no\&accno=EJ874205

Nicaud, Jean-François, Bouhineau, Denis, Varlet, Christian y Nguyen-Xuan, Anh. (1999) Towards a product for teaching formal algebra. Presentado en Artificial Intelligence in Education, Le Mans, Francia. Recuperado de http://halshs.archives-ouvertes.fr/ docs/00/19/03/02/PDF/Bouhineau-Denis-1999.pdf

Ritter, Steven, Anderson, John, Koedinger, Kenneth y Corbett, Albert. (2007). Cognitive tutor: applied research in mathematics education. Psychonomic
Bulletin \& Review 14(2), 249-255. Recuperado de http://pact.cs.cmu.edu/pubs/Ritter\%20Anderson\%20 Koedinger\%20Corbett\%202007.pdf.

Sierra Tortosa, Guillermo. (2010). Didáctica del álgebra. Revista Digital I+E Investigación y Educación (26). Recuperado de http://www.csi-csif.es/andalucia/modules/mod_ense/revista/pdf/Numero_26/ GUILLERMO_SIERRA_TORTOSA.pdf

Tobón, Sergio. (2004). Formación basada en competencias. Pensamiento complejo, diseño curricular y didáctica. Bogotá, ECO.

Tobón, Sergio. (2008). La formación basada en competencias en la educación superior. Curso Iglu, Universidad Autónoma de México, Guadalajara, México. Recuperado de http://www.conalepfresnillo. com/images/stories/conalep/Formaci\%C3\%B3n\%20 basada $\% 20$ en $\% 20$ competencias. $\% 20$ Sergio $\% 20$ Tob\%C3\%B3n.pdf

Vergnaud, Gérard. (1990). La théorie des champs conceptuels, Recherches en Didáctique des Mathématiques 10(2), 133-170. Versión en español recuperada de http://fundesuperior.org/Articulos/Pedagogia/Teoria_ campos_conceptuales.pdf. 
